\title{
Drug Prescription Modifies the Doctor-Patient Relationship in General Medicine
}

\author{
Jose Luis Turabian* \\ Specialist in Family and Community Medicine, Health Center Santa Maria de Benquerencia, Regional Health Service of \\ Castilla la Mancha (SESCAM), Spain
}

\begin{abstract}
It is within of the doctor-patient relationship is where, very often, the decision to prescribe a drug occurs. It is classically accepted that the general practitioner is itself a drug. So, the doctor-patient relationship itself can have a therapeutic action. The consultation relational context that the doctor creates in his patient care can function as a drug. This therapeutic context induces biomedical processes in the patient's brain that may enhance or reduce the effects of chosen interventions. In this way, when the general practitioner prescribes a drug, he should modify his "psychotherapy" or his advice according to the pharmacological treatment of the patient. The drugs act on the symptoms and change thoughts, feelings and behaviors (not only the psychotropic drugs; any drug); they can create both physical and psychological dependence, whose effect can be mediated by biochemical and/or psychosocial factors; they can discourage a deep search for real solutions, both by the doctor and the patient; they can affect general practitioner access on the patient and the problem will be out of reach of him. The drugs can make the effect of the "doctor itself as a drug" can be more difficult, favouring a relational context that is not significant or that be problematic or not very human, where the general practitioner does not delve into the true meaning of the symptoms, and the patient tends not to get involved, to performing an emotional withdrawal, to be passive before the prescribed drug, and all of this can bring as a consequence, chronification, structuring of functional symptoms that become organic.
\end{abstract}

\section{Keywords}

Physician-patient communication, Pharmaceutical treatment, Therapeutic alliance, Inappropriate prescribing, General practice, Framework, Decision making, Therapeutic adherence, Physician-patient relations, Sanitary attention

"I don't do drugs. I am drugs". - Salvador Dali [(11 May 1904 - 23 January 1989), was a prominent Spanish surrealist painter].

"I think the smartest thing for people to do to manage very distressing emotions is to take a medication if it helps, but don't do only that. You also need to train your mind". - Daniel Goleman [(Born March 7, 1946) is an author and science journalist].

\section{Introduction}

The doctor-patient relationship is a special type of human relationship that occurs in the clinical encounter between doctor and patient [1]. Probably one of the first studies on the doctor-patient relationship was carried out by Freud [2]. From that moment, and exponentially in recent times, analyzes on this subject follow one another.

It is in the heart of the encounter and of the doctor-patient relationship where the diagnosis is made and the treatment is decided, and the doctor-patient relationship is fundamental so that the doctor can make the diagnosis and the treatment $[3,4]$. And so, it is within that relationship where, very often, the decision to prescribe a drug occurs. Probably this situation is especially more frequent in general medicine, where in addition to the prescriptions as the end of a proper diagnosis, there are the repeated prescriptions of chronic problems or of repeated requests of the patients, as well as the so-called derived prescriptions, prescriptions made by another doctor different from the general practitioner (GP), usually a hospital consultant, and who refers the patient to his GP to repeat medicines.

In this scenario, where there is a certain lack of agreement in the way of understanding and classifying the different types of doctor-patient relationship, we are missing a current

*Corresponding author: Jose Luis Turabian, Specialist in Family and Community Medicine, Health Center Santa Maria de Benquerencia, Regional Health Service of Castilla la Mancha (SESCAM), Toledo, Spain, ORCID iD: 0000-0002-8463-171X

Accepted: November 22, 2018

Published online: November 24, 2018

Citation: Turabian JL (2018) Drug Prescription Modifies the Doctor-Patient Relationship in General Medicine. Arch Fam Med Gen Pract 3(1):66-69 
reflection on the interference or modification that can be made by the prescription of a drug, which is something usual in the consultations, on the relationship doctor-patient.

\section{Discussion}

It is classically accepted that the GP is itself a drug; He is the "doctor"-drug. The doctor-patient relationship itself has a therapeutic action. In other words, the relational context that the doctor creates in his patient care is in itself also a drug. The relationships and communications that he establishes with the patients in the consultation function as a drug. The "creation of context" is the result of the doctor-patient relationship strategies in the course of the clinical interview for diagnosis: Whether it is a paternalistic communication, or persuasive or centered on the patient, etc., these strategies are "creators of a therapeutic or anti-therapeutic context" [5].

Sometimes, the relationship between the doctor and the patient is difficult or problematic; in these cases the "drug"-doctor does not achieve the expected results. This medicine called "doctor" is powerful and can have many side effects. You have to know how to dose and prescribe this "drug"-doctor in this context where the pharmacological prescription is produced [6]. It has been reported that this therapeutic context induces biomedical processes in the patient's brain that may enhance or reduce the effects of chosen interventions. The context thus works as a drug, with real effects and side effects [7], and we must remember that, the nocebo-placebo effect is also included here $[8,9]$.

A pharmacological prescription whose decision is expressed by the doctor with great security, usually has a placebo effect; On the other hand, if the decision is considered to be of doubtful effect, it will frequently give rise, depending on the general context of the consultation and the personality characteristics of the patient, to a nocebo effect, with poor therapeutic results or adverse pharmacological effects (which are not chemically mediated, but psychosocially) [10-14].

In this way, when the general practitioner prescribes a drug, he should modify his "psychotherapy" or advice according to the pharmacological treatment of the patient; it is not appropriate to apply the same techniques -cognitivebehavioral in general- both patients without drugs and with drugs [12]. There is a well-established approach in medicine that says that if the symptoms of a disease are effectively eliminated, it can be assumed that the disease is being treated. This approach leads physicians towards symptomatic treatment, which is more accessible and tangible than the patient's internal experience. Obviously, this vision is more typical of the mental health sphere, but it can rationally be extended to all the health problems addressed by GP.

Emotional distress leads to biochemical changes in the brain and thus thoughts, feelings and behaviors are biochemically mediated. However, the medical model assumes that it is the other way around, and that changes in thoughts, feelings and behaviors cause biochemical changes, and therefore, pharmacological treatment will lead to improved distress. This bilateral relationship between distress and chemistry assumes that the external factors of life experience, relationships and events could be mitigated by the change in the patient's psychological state.

For example, a person with a certain range of symptoms can take some kind of steps to avoid them. Drugs change brain chemistry, decrease symptoms, reduce anxiety, and consequently affect the thoughts, feelings and behaviors of the person. It is assumed that the person can therefore control their problem more effectively once their symptoms have been eliminated. The underlying assumption is not that the symptoms are an inappropriate or undesirable state, but that they do not have a deep meaning.

However, in the biopsychosocial model, which is the theoretical framework of general medicine, it is assumed that the symptoms are something related to the person's life. In addition, from this perspective the disorder itself can be therapeutic, since the appearance of the signs of distress, such as symptoms and changes in mood and behavior that are provoked in the individual make him adjust his understanding of the self, his interpersonal relationships, or his life.

Evidently, these models lead to different conclusions about therapeutic options. In the medical model, the general practitioner sees as a mandatory intervention the prescription of one or more drugs, and as an objective of pharmacological treatment the resolution of the problem or distress. In the bio-psycho-social model, GP recognizes that feelings of emotional distress may be appropriate, unavoidable and even acceptable especially for a limited time, and thus not prescribe drugs.

The approach to the epidemiological chain of causality, in the medical model, is about the biochemical mechanisms through which all biopsychological processes are mediated, and the disease is seen as an imbalance that must be restored with drugs and leads to the idea that some people have to take drugs for the rest of their lives to maintain this balance. It also leaves aside the need to confront the internal psychological reality. The patient who has been treated with drugs and their symptoms have been relieved, will be more reticent, or will be less motivated to look for real and possibly more painful solutions. Giving benzodiazepines for insomnia will make finding the cause of insomnia more difficult. And so, this is true in a great variety, but in all the symptoms.

In general terms, the pharmacological treatment is aimed at changing the biochemistry and the symptoms and consequently the emotions, while the counselling that is produced from an adequate doctor-patient relationship, works from the inner world of the patient to change the experience and perception about their health problem, and consequently eliminate the symptoms.

The symptoms suppose a focus of attention since they indicate in some way the inner conflict or the evidence that the defensive systems have been activated. Symptoms are also the way in which the patient defines their problem: A way to make something accessible or available to someone. The patient can begin by describing his physical symptoms instead of saying how he feels. Frequently physical symptoms can be expressed through metaphors even if the patient is not fully aware of it. Symptoms occur in sets that collectively have 
a special meaning. If the symptoms can be easily understood in terms of being caused or related to some experience or event, they can be tolerated even if they are severe.

Understanding the meaning of the symptoms provides to the GP with a way to access the problem that the patient brings. The symptoms take meaning in the context, and can be seen as a silent form of communication with others. It may be important for the patient to cling to their symptoms as a way of defining themselves, and until a broad sense of self is established, when we try to eliminate the symptoms, they may even get worse. This is the reason why sometimes the drugs, which are a symptomatic treatment, often make the situation worse. GPs who work with patients who take drugs need to be more active to allow access to patients' feelings.

On the other hand, in some diseases, the prescription of drugs affects the relationship that the patient has with his illness and with the doctor, since it allows him a certain gain as well as a co-expert, as well as being able to give name and status of reality about his symptoms (they already have value for the doctor; their complaints are believed by doctor; so they are treated with drugs), as it can happen in fibromyalgia [15].

Another interesting aspect is the repeated prescription of drugs, in chronic problems or by reiteration of the patient's demand or by prescription derived from a hospital consultant, and performed by computer or electronic means, specifically, that modifies the doctor-patient relationship. This type of relationship symbolizes a "failure", a continuity "peaceful but without hope", which maintains a status quo, but where both doctor and patient are defeated. It is a "tolerable but cold" doctor-patient relationship that lasts for years, but with the conflicts of the problem always latent. Here, the effect of the doctor himself as a drug is very small, "homeopathic", or almost nonexistent; the patient only goes to "repeat his prescription", not to make a consultation. In addition, the patient usually considers their drugs repeated as "their right". Any change of drugs, dose modification or withdrawal is experienced in a problematic way. All this modifies the doctorpatient scenario and makes it difficult for the interpersonal relationship can go beyond the routine superficial [6].

In this scenario, in addition, a dramatic and incorrect transformation of the doctor-patient relationship in general medicine is taking place. When priority is given to the prescription of drugs vs. the interpersonal relationship (or in other words, the biomedical vs. the biopsychosocial model) in general medicine, the doctor-patient relationship becomes exclusively in a pharmacological relationship; this is, from the doctor himself as a drug to doctor as drug dealer, where the doctor himself as a drug is excluded. This model of impersonal care can be accepted willingly by the GP, because medications are used to avoid being with people, and being a doctor as a drug dealer is easier than showing us human [16].

All these forces act on the patient, and thus, the GP may suspect that some patients value the quality of their medical care for the amount of pharmacological prescriptions they receive: "the more medications they receive, the happier they are". And it seems that patients who receive antibiotic prescriptions are the most satisfied. In this way, it can be thought that many patients expect drugs from a medical visit. It also suggests that doctors need to master the essential art of persuading patients that they do not need unnecessary medications, which can be an clinical interview more difficult than prescribing drugs [17].

\section{Conclusion}

In summary, that the GP understands the psychosocial effects of the prescription in the doctor-patient relationship is as important if not more than to know the pharmacology. The drugs act on the symptoms and change thoughts, feelings and behaviors; they can create both physical and psychological dependence; they can discourage a deep search for real solutions, both by the doctor and the patient; they can affect GP access on the patient and the problem will be out of reach. The drugs can make the effect of the "doctor itself as a drug" can be more difficult, favouring a relational context that is not significant or problematic or not very human, where the GP does not delve into the true meaning of the symptoms, and the patient tends not to get involved, perform an emotional withdrawal, to be passive before the prescribed drug, and can bring as a consequence, chronification, structuring of functional symptoms that become organic, lack of cooperation of the doctor and the patient, and paradoxically the over-compliance or therapeutic discontinuity and lack of pharmacological adherence, absences to appointments or delays or cancellations, and denial of the responsibility of both doctor and patient.

\section{References}

1. Pellegrino ED (2001) The internal morality of clinical medicine: A paradigm for the ethics of the helping and healing professions. J Med Philos 26: 559-579.

2. Pérez Cicili A, Vidaillet Calvo EC, Carnot Pereira J, et al. (2003) The doctor-patient relationship in the national health system. Medicina General Integral 19.

3. Turabián Fernández JL, Pérez Franco B (2010) The concept of treatment in family medicine: A contextualised and contextual map of a city hardly seen. Aten Primaria 42: 253-254.

4. Turabián Fernández JL, Pérez Franco B (2008) The effect of seeing the sea for the first time. An attempt at defining the family medicine law: The interview is clinical medicine. Primary Care 40: 565-566.

5. Turabián JL, Pérez Franco B (2001) Community activities in family medicine and primary care. Díaz de Santos, Madrid.

6. Balint M, Hunt J, Joyce D (1984) Treatment or diagnosis. A study of repeat prescriptions in general practice. Tavistock Publications, London.

7. Lucassen P, Olesen F (2016) Context as a drug: Some consequences of placebo research for primary care. Scand J Prim Health Care 34: 428-433.

8. Leigh H, Reiser MF (1992) The patient. Biological, psychological, and social dimensions of medical practice. Plenun Medical Book Company, New York.

9. Turabian JL (2018) Contextual treatment: A conceptualization and systematization from general medicine. Int J Fam Commun Med 2: 97-104. 
10. Gutiérrez-Islas, Báez-Montiel BB, Turabian JL, et al. (2012) Patients with adverse drug reactions have a higher prevalence of emotional disorders. Primary Care 44: 720-726.

11. Turabian JL, Moreno-Ruiz S (2016) The fable of the pine and the palm tree: The two extremes. Strategies to maximize the placebo effect and minimize the nocebo effect in primary health care. Ment Health Addict Res 1: 44-46.

12. Hammersley D (1995) Counselling people on prescribed drugs. Sage Publications, London.

13. Faasse K, Petrie KJ (2013) The nocebo effect: Patient expectations and medication side effects. Postgrad Med J 89: 540-546.

14. Evers AWM, Colloca L, Blease C, et al. (2018) Implications of placebo and nocebo effects for clinical practice: Expert consensus. Psychother Psychosom 87: 204-210.

15. Durif-Bruckert C, Roux $P$, Rousset $H$ (2015) Medication and the patient-doctor relationship: A qualitative study with patients suffering from fibromyalgia. Health Expect 18: 2584-2594.

16. Turabian JL (2018) The wrong transformation of doctor-patient relationship in drug-patient relationship: From the doctor himself as a drug to doctor as drug dealer. Chronicle of Medicine and Surgery 3: 298-301.

17. Martinez KA, Rood M, Jhangiani N, et al. (2018) Association between antibiotic prescribing for respiratory tract infections and patient satisfaction in direct-to-consumer telemedicine. JAMA Intern Med 178: 1558-1560. 